\title{
Consecuencias sociales y culturales de la sobreeducación ${ }^{1}$
}

\section{Rafael Gobernado Arribas}

Universidad de Málaga

gobernado@uma.es

\section{Resumen}

A partir de una definición operativa de sobreeducación, hemos clasificado sus consecuencias para la población española en tres categorías: consecuencias empresariales u organizacionales, consecuencias privadas y consecuencias de carácter social y político. En líneas generales, la sobreeducación no tiene consecuencias muy acusadas en los ámbitos indicados, sino que parece compensarse de diferentes maneras. La compensación principal en el ámbito organizacional es la esperanza de promoción; en el ámbito privado, se compensa con una mayor participación en actividades de ocio y aficiones. Donde se vuelca más la insatisfacción es en el ámbito público: la persona sobresocializada es más radical en su ideología y está más politizada que el resto de la población.

Palabras clave: educación, empleo, organización, empresa, ideología.

\section{Abstract. Social and cultural consequences of over-education}

Starting from an operational definition of over-education, we have classified its consequences for the Spanish population into three categories: professional or organizational, private and socio-political consequences. Generally speaking, over-education does not have very marked consequences on the above mentioned fields (areas), but it seems to compensate itself in different ways. In the professional area, it mainly influences the hope of promotion; in the private field, it makes over-educated people take a more active part in leisure (free time) activities and hobbies. It is in the public field where dissatisfaction is more noticeable: over-socialized people are ideologically more radical and more politicized than the rest of the population.

Key words: education, employment, organization, company, ideology.

1. Este trabajo se ha podido realizar gracias a la colaboración del Centro de Estudios Andaluces: proyecto SOC-6 del año 2005. 


\section{Sumario}

1. Objetivos y antecedentes

2. El método

3. Sobreeducación, trabajo y empresa u organización

4. Consecuencias privadas de la sobreeducación
5. Consecuencias sociales y políticas de la sobreeducación

6. Conclusiones

Bibliografía

\section{Objetivos y antecedentes}

La finalidad de este trabajo consiste en establecer y explicar las relaciones entre sobreeducación, por un lado (variable independiente), y un conjunto de variables sociales y culturales de diversa índole, por otro (variables dependientes). Estas variables dependientes han sido clasificadas en tres categorías: variables que tienen que ver con la organización o la empresa en la que se trabaja; variables propias de la vida privada del entrevistado, y variables de carácter político o colectivo. Por supuesto, los tres tipos de variables se encuentran interrelacionados y la clasificación sólo es un artificio para facilitar el acceso al análisis en cuestión. El análisis empírico utiliza como base de datos las encuestas de calidad de vida en el trabajo, del Ministerio de Trabajo y Seguridad Social, de los años 2003 y 1999. Los resultados, por lo tanto, sólo son generalizables al mercado laboral español de tales fechas.

El principal problema de la presente investigación es definir el concepto de «sobreeducación». Existe sobreeducación cuando la población que constituye la demanda laboral posee un nivel educativo formal superior al que exige la oferta de empleo. Sobreeducación es sinónimo, por lo tanto, de infraocupación. Como se sabe, la educación (y también la sobreeducación) es un concepto diferente al de formación en el puesto de trabajo: la educación se adquiere en organizaciones educativas al efecto y la formación se adquiere en las empresas u organizaciones en las que se trabaja. Mientras que el ensamblaje entre formación y empleo es natural y espontáneo, el ensamblaje entre educación y empleo es más bien convencional ${ }^{2}$. La sobreeducación se produce no sólo en el acceso al mercado laboral o primera ocupación, sino también en la propia estructura del mercado de trabajo. Es decir, tiene lugar en cualquier edad de la población ocupada.

El indicador más claro de sobreeducación es la pérdida comparativa de rentabilidad económica (ingresos) y social (prestigio) del esfuerzo educativo. Tal situación se explica en este trabajo fundamentalmente desde la teoría del logro ocupacional $^{3}$ : todos deseamos mejorar de posición social (movilidad social).

2. Sobre las dificultades de ensamblar educación y empleo, véase C. Béduwé y J. Planas (2002: 62-68).

3. Las explicaciones del proceso de sobreeducación son muy variadas. Para una visión general del tema, véase R. Gobernado (2005). 
Ahora bien, la mejora de la posición social depende de factores controlables por el actor (educación, esfuerzo, por ejemplo) y de circunstancias no controlables (origen social, mercado laboral, oferta educativa, entre otros). Es decir, el nivel educativo es un factor que controla (relativamente) el individuo y que sirve para prosperar en la posición social. Por lo tanto, hay un claro interés en aumentar el nivel de la misma, sobre todo por encima del nivel de los demás, lo que produce un proceso de sobreeducación ilimitado, en principio. Ésta es también una de las teorías favoritas de los economistas para explicar la sobreeducación (véanse, por ejemplo, P. Skott, 2005; O. Charlot y B. Decreuse, 2005; I. Kogan y M. Unt, 2005; F. Buchel y A. Mertens, 2004). En sociología, por el contrario, parece que tienen más aceptación las teorías del capital cultural (P. Bourdieu) y del credencialismo (R. Collins) (véase, B.-H. Burris, 1983).

Cuando el individuo se encuentra en una situación de sobreeducación, es decir que no ha conseguido ese logro ocupacional que preveía, caben varias consecuencias. Posiblemente, la persona sobreeducada se encuentra incómoda en su trabajo y a) transmite esa incomodidad a la organización en la que presta sus servicios o busca salidas en la misma; $b$ ) privatiza la incomodidad que siente en el puesto de trabajo, es decir, tiende a estar insatisfecho con otros aspectos de su vida mas allá del mundo laboral o, por el contrario, quita importancia al trabajo y se lo da a otras condiciones ajenas al mismo entre las que se encuentra más a gusto, y $c$ ) proyecta su descontento y la compensación consecuente en el ámbito social y político del entorno ${ }^{4}$.

También cabe otra posibilidad: la persona no aprecia claramente esa situación de sobreeducación. Esto es posible porque nos movemos con elementos sistémicos (educación, empleo, ingresos, etc.) muy fluidos, en constante búsqueda de equilibrio, y cuya difícil percepción subjetiva impide desarrollar ese sentimiento de incomodidad, al menos a corto plazo. Más concretamente: la construcción teórica de nivel micro (basada en la racionalidad individual y en que todos queremos una posición social mejor) tiene que ver, a su vez, con una construcción macro que denominamos modernización, es decir, la organización social moderna se caracteriza por un crecimiento paulatino de la incoherencia entre los indicadores sociales. Así, por ejemplo, la clase social de origen tiene menos que ver con la clase de pertenencia (mayor movilidad social y espacial), a su vez, ésta se relaciona menos con el nivel educativo y ambas tienen que ver también menos con los ingresos, etc. O lo que es igual, se trata de un proceso según el cual el comportamiento individual se va independizando cada vez más de las categorías sociales de pertenencia. La dirección de voto, la práctica religiosa, la ideología en general dejan de depender de la clase social, el nivel educativo, la edad, el sexo, etc. En unas circunstancias de este tipo, se acaba por aceptar como normal cualquier incoherencia social, incluida la

4. Esta clasificación está en deuda con la clásica de K. Polanyi (1976) de pautas económicas de integración: a) las basadas en el intercambio (en nuestro caso se trata del mercado laboral), b) las basadas en la reciprocidad (las del ámbito privado), y $c$ ) las basadas en la redistribución (las propias del estado). 
que se da entre educación y ocupación. (Sobre los cambios hacia la modernidad que influyen en esta situación, véanse, por ejemplo, M. H. J. Wolbers y otros, 2001; R. Gobernado, 1996a y 1996b.)

Como suele ocurrir en las ciencias sociales, todas las explicaciones son admisibles y se complementan. Posiblemente, no obstante, gran parte de la inocuidad aparente de la sobreeducación tiene que ver con la última posibilidad, la del enfoque macro de la modernización: la sobreeducación se vuelve opaca en el contexto general de la modernización.

La sospecha de que los diferentes indicadores de desigualdad social no tienen por qué ser congruentes, es antigua. Ya Max Weber, en Economía y sociedad (1979: 245), apuntó la idea de que la situación económica (la clase social) no coincidía necesariamente con el prestigio (o estatus) ni con el grado de poder. No obstante, la idea de la falta de relación entre los indicadores de desigualdad toma forma sobre todo a partir del artículo de G. Lenski de 1954 (y también 1956, 1967) sobre "la cristalización de estatus». La idea que desarrolla es muy interesante: la congruencia (o falta de la misma) de indicadores de desigualdad social en una persona puede explicar su comportamiento tanto o más que la pertenencia a un estrato o clase social. Lenski afirmaba, por ejemplo, que la dirección de voto hacia el radicalismo político o hacia el liberalismo tenía que ver con la ausencia o la presencia, respectivamente, de tal congruencia entre indicadores. Desde entonces, se ha sucedido una gran cantidad de estudios que han desarrollado la idea original por múltiples direcciones.

El estudio de la influencia de la sobreeducación en el actor social es una forma más de desarrollar la idea de Lenski: se entiende por sobreeducación a la inconsistencia lógica entre la preparación educativa del empleado y las necesidades educativas del puesto de trabajo. Como luego se verá, en nuestro caso el desajuste se produce entre el nivel educativo y el prestigio de la ocupación laboral. En la actualidad, el nivel educativo de la demanda de empleo es muy superior a las necesidades de la oferta. La mayor parte de la población empleada tiene un nivel educativo formal muy por encima de las necesidades del mercado laboral. Esta situación es de carácter general. Sin embargo, cuando aquí hablamos de sobreeducación, nos referimos exclusivamente a la sobreeducación relativa, no a la general. Se entiende por sobreeducación relativa aquélla que afecta a una minoría dentro de cada categoría laboral. Según esto, unos pocos dentro de cada categoría laboral tienen un nivel educativo muy superior al resto de los ocupados en la misma categoría.

Los estudios descriptivos de sobreeducación tienen ya cierta antigüedad: aparecen síntomas de sobreeducación en Estado Unidos en los años cuarenta y cincuenta (J. K. Folger y Ch. B. Nam, 1964) y continúa en los sesenta (I. Berg, 1970) y setenta (R. Rumberger, 1981). La bibliografía sobre el tema se multiplica. P. J. Sloane (2003) ofrece una visión panorámica de estos estudios descriptivos en Europa y Estados Unidos (para más información sobre Europa, véanse F. Büchel y otros, 2003; C. Béduwé y J. Planas, 2002).

Además de la simple descripción, surge también el interés por sus consecuencias. Desde un primer momento, se pensó que la sobreeducación causaba 
insatisfacción con el trabajo (I. Berg, 1970) y posturas izquierdistas radicales (A. Gorz, 1967). En resumen, generaba descontento político y social. Más recientemente, V. Burris (1983) observó que la sobreeducación en Estados Unidos apenas generaba insatisfacción en el trabajo, ni tendencias radicales hacia la izquierda, ni alienación política. Según este autor, en Estados Unidos, la consecuencia más probable de la sobreeducación era la progresiva "privatización del descontento», lo cual facilitaba la aparición de sentimiento de culpa (baja autoestima, estrés, etc.) o también una adaptación individual a través de una redefinición del estatus (se daba más importancia a la familia y al ocio, que al trabajo). Otros trabajos más actuales defienden también la idea de que la sobreeducación no es negativa, ni para la empresa, ni para el que la soporta (F. Buchel, 2002).

La presente investigación tiene como objetivo ver si la sobreeducación en España tiene consecuencias culturales (ideacionales) y sociales y, en su caso, si estas consecuencias son poco o muy acusadas. Como se dijo arriba, se ha clasificado las consecuencias en tres tipos: $a$ ) consecuencias organizacionales; $b$ ) consecuencias personales (privadas, según la terminología de V. Burris), y c) consecuencias sociales y políticas. Cabe la posibilidad, por último, de que la persona que sufre sobreeducación no tome conciencia de la misma o no le preste importancia. Para ello, nos apoyamos en las encuestas de calidad de vida en el trabajo, la del año 2003 y, en menor cantidad, en la de 1999, elaboradas por el Ministerio de Trabajo y Seguridad Social.

\section{El método}

Las formas habituales de contabilizar la sobreeducación (P. J. Sloane, 2003: 15-20) plantean problemas (R. Gobernado, 2005). La denominada forma objetiva depende de un registro oficial de ocupaciones que no existe en nuestro país. La forma empírica (dispersión por encima de la media de años de escolaridad en cada categoría profesional, más allá de una desviación estándar) no da resultados apropiados, ya que la población sobreeducada según este criterio también tiene ingresos por encima de la media y no hay sobreeducación si el nivel educativo ayuda a ganar más dinero, como suele ocurrir (véase $S$. Rubb, 2003). Y el criterio subjetivo (preguntar al actor si se considera o no sobreeducado) tampoco sirve, ya que el entrevistado suele informar sobre la sobreeducación general y no sobre la relativa: según esto, la gran mayoría de los entrevistados está sobreeducada. El poco interés de estos criterios se aprecia en que apenas hay correlación entre los tres (H. Battu y otros, 2000).

Para facilitar nuestra tarea, hemos utilizado otra fórmula de contabilidad de la población sobreeducada. El punto de partida ha sido crear una escala de cinco estratos sociales. Para ello, se ha aplicado la escala de prestigio social de J. Carabaña y C. Bueno (1996) a los datos sobre ocupación de la encuesta. El continuo de la escala de prestigio se dividió, a su vez, en cinco estratos. En cada estrato se ubica el 20 por ciento aproximado de la población entrevistada. Los estratos van desde el superior (1) hasta el inferior (5). El siguiente paso fue cla- 
sificar la población encuestada según cuatro niveles educativos: analfabetos y sin estudios; primarios; medios, y superiores. Los estudios superiores están formados por licenciaturas, diplomaturas y doctorados.

A partir de las dos variables anteriores, hemos establecido dos escalas de sobreeducación. Según la primera (sobreeducación 1), se ha limitado el análisis a la población con estudios superiores (diplomados, licenciados y doctores) repartidos en los cinco estratos de prestigio ocupacional. El análisis de esta variable supone un continuo de sobreeducación de cinco grados, desde el máximo (la población con estudios superiores que trabaja en ocupaciones de prestigio social inferior), hasta el mínimo (población con estudios superiores que trabaja en ocupaciones propias del estrato superior).

La segunda escala de sobreeducación (sobreeducación 2) es complementaria de la anterior. En este caso, se limita el análisis a los que realizan trabajos ubicados en los dos estratos inferiores, los de menor prestigio social, distribuidos según el nivel de estudios. En este caso, el continuo tiene cuatro grados y va desde el máximo de sobreeducación (estudios superiores en los dos estratos sociales inferiores) hasta el mínimo (sin estudios en los dos estratos inferiores).

En ningún caso estas escalas sirven para describir el estado de sobreeducación de una población determinada, ya que no alcanzan a la totalidad de la población entrevistada, sino únicamente a una proporción de la misma. Son útiles solamente para establecer relaciones explicativas con otras variables de la investigación, las dependientes. Ahora bien, su uso plantea el problema de la complementariedad entre las dos definiciones. La jerarquía de prestigio tiene mucho más que ver con la varianza de las variables dependientes que la jerarquía educativa. Así, por ejemplo, la posición social del puesto de trabajo tiene más que ver con los ingresos que el nivel educativo (R. Gobernado, 2003). Entiendo, por ello, que la segunda forma de definir la sobreeducación (jerarquía educativa de los estratos ocupacionales inferiores) es más correcta que la primera. En ésta, la sobreeducación 1, la diferencia de estrato se sobrepone a la diferencia de nivel educativo. La sobreeducación primera tiene más que ver con la diferencia de estratos que con la propia sobreeducación. Su capacidad explicativa oscurece la capacidad explicativa de la sobreeducación. Esto no ocurre con la segunda fórmula, en la que el nivel educativo cobra protagonismo y, por lo tanto, la sobreeducación también (sobre los inconvenientes y ventajas de ambas formas, véase S. Rubb, 2005).

Estas dos escalas de sobreeducación constituyen las variables independientes del análisis. Las variables dependientes se definen, en su momento, posteriormente. Están formadas por índices de escalas tipo Likert, a partir de conjuntos ya elaborados de la Encuesta de calidad de vida en el trabajo y por variables simples cuya sintaxis es de tipo Cantril (valores entre 1 y 10 , o entre 1 y 5 , como se verá). Algunas variables, pocas, son de carácter nominal o cualitativo. En general, la mayoría de ellas son de carácter ideacional (cultural o psicosocial). Así mismo, se tiene en cuenta una serie de variables externas que se controlan en el análisis (tales como edad, tamaño del hábitat, tamaño de la empresa, tipo de contrato laboral, ingresos, etc.). 
Para detectar el conjunto de relaciones, se han realizado dos tipos de análisis. Por un lado, análisis de medias cuyo resultado principal es la prueba de la $F$ (comparar la varianza intra grupos y extra grupos) así como obtener, en su caso, el coeficiente de determinación a partir de la R-cuadrado (véase J. J. Sánchez Carrión, 1995: 339-341). El segundo tipo de análisis ha sido el de regresión lineal, que nos permite apreciar mejor el peso de las variables externas.

Las bases de datos utilizadas son las encuestas de calidad de vida en el trabajo de los años 2003 y 1999, realizadas por el Ministerio de Trabajo y Seguridad Social en el mes de junio de esos mismos años. La muestra de cada una está formada por 6.000 personas ocupadas y ha sido ponderada por los propios autores de la encuesta según sexo, edad, comunidad autónoma y tamaño del municipio. Representa, por lo tanto, bastante acertadamente a la población ocupada española de los citados años. De cualquier forma, el uso de esta base de datos impide entrar en detalle y particularismos que harían el análisis más interesante.

\section{Sobreeducación, trabajo y empresa u organización}

El objetivo inmediato ahora es apreciar si las personas sobreeducadas desarrollan una forma particular, diferente, de entender la relación laboral con la empresa u organización en la que trabajan. Para ello, hemos utilizado como variables dependientes las variables siguientes, todas ellas previstas en la Encuesta de calidad de vida en el trabajo:

1. Satisfacción en el trabajo ${ }^{5}$ (muy insatisfecho $=1$; muy satisfecho $=10$ ).

2. Interés por el propio puesto de trabajo $^{6}$ (mucho interés $=5$; ningún interés $=1)$.

3. Interés por la empresa u organización ${ }^{7}$ (mucho interés $=5$; poco interés $=1)$.

5. Escala tipo Cantril que oscila entre 1 (muy insatisfecho) y 10 (muy satisfecho).

6. Escala tipo Likert formada por cinco grados que oscilan entre el total acuerdo (5) y el total desacuerdo (1) con estas siete proposiciones: «mi trabajo es atractivo e interesante», «puedo trabajar con independencia y poner en práctica mis ideas», «en mi trabajo, puedo ayudar a la gente», «mi trabajo es útil a la sociedad», «mi empleo es estable», «el entorno físico en el que trabajo es agradable» $y$ «siempre participo en las decisiones respecto a las tareas a realizar en mi trabajo».

7. Escala tipo Likert formada por cinco grados que oscilan entre el total acuerdo (5) y el total desacuerdo (1) con las nueve proposiciones siguientes: «estoy dispuesto a trabajar más de lo que debo para ayudar a triunfar a la empresa u organización para la que trabajo», «en mi empresa/organización existe colaboración porque hay una jerarquía que hace que se cumplan las tareas», «estoy orgulloso de trabajar en mi empresa u organización», «si tuviese la oportunidad, cambiaría mi trabajo actual por algún otro diferente», «si pudiera, me establecería por mi cuenta abriendo mi propio negocio, o como autónomo», «en el trabajo, lo importante es cumplir con las obligaciones y actividades que establece el contrato, extralimitarse significa que, al final, no te lo agradecen ni sirve de nada», «los problemas que tiene mi empresa u organización los considero como si fueran míos» $\mathrm{y}$ "en realidad, sólo se puede confiar en la familia». 
4. Actitud ante el trabajo en general $^{8}$ (muy positiva $=5$; muy negativa $=1$ ).

5. Condiciones del trabajo9 (muy duras $=5$; muy buenas $=1$ ).

6. Alienación respecto al trabajo ${ }^{10}($ mucha $=5$; ninguna $=1)$.

7. Confianza con el entorno laboral ${ }^{11}$ (mucha $=5$; ninguna $\left.=1\right)$.

8. Conocimiento de la organización ${ }^{12}($ mucho $=5$; ninguno $=1)$.

9. Posibilidades de promoción $(1=\text { ninguna; } 5=\text { muchas })^{13}$.

10. Cambio de empleo ( 1 = seguramente, sí; 5 = seguramente, no $)$.

11. Situación de pluriempleo (sí / no).

Sabemos que el interés de estas variables en el éxito o fracaso de las empresas es relativo, más bien exiguo (J. A. Garmendia, 2004). El caso paradigmático es el de la satisfacción en el trabajo, puesto que se llegó a pensar incluso que ésta facilita más los malos resultados empresariales que los buenos (E. L. Lawler y L. W. Porter, 1967). De cualquier forma, la influencia en la buena marcha de la empresa de estas variables no es el tema que nos ocupa ahora, sino la relación entre el exceso de educación y esas variables ideacionales indicadas arriba.

El resultado del análisis de medias de las variables dependientes citadas según las dos formas de definir la sobreeducación, se expone en la tabla 1 . De los datos se deduce, ante todo, que la diferencia de estratos (definición 1 de sobreeducación) influye mucho más en las variables consideradas que la dife-

8. Escala tipo Likert formada por cinco grados que oscilan entre el total acuerdo (5) y el total desacuerdo (1) con estas cinco proposiciones: «un empleo es una forma de ganar dinero y nada más», "me gustaría tener un trabajo remunerado, aunque no necesitase el dinero», «el trabajo es la actividad más importante de cualquier persona», «es el tiempo libre, y no el trabajo, lo que hace que valga la pena vivir» y «en mi empresa/organización las personas que trabajamos juntas confiamos unas en otras, porque así se hace el trabajo más fácil y mejor».

9. Escala tipo Likert formada por cinco grados que oscilan entre siempre (5) y nunca (1) respondiendo a las cuatro preguntas siguientes: «¿tiene que hacer grandes esfuerzos físicos en su trabajo?», "¿considera que su trabajo es estresante?», "¿trabaja Vd. en condiciones peligrosas?" y "¿le facilita su empresa o se provee $\mathrm{Vd}$. mismo si es autónomo de los medios necesarios para trabajar en condiciones seguras?».

10. Escala tipo Likert formada por cinco grados que oscilan entre siempre (5) y nunca (1) según la ocurrencia de los siguientes pensamientos: «el tiempo se me va muy rápido», «la jornada laboral se me hace aburrida y monótona», «me distraigo pensando en las cosas que considero importantes, como la familia», "vuelvo a casa muy cansado del trabajo», «tengo flexibilidad en decidir cuando llegar o cuando marcharme» y «el horario que tengo lo determino yo mismo».

11. Escala tipo Likert formada por cinco grados que oscilan entre mucha confianza (5) y nada (1) en: "confianza en mis superiores», "confianza en mis compañeros» $y$ «confianza en mis subordinados».

12. Escala tipo Likert formada por cinco grados que oscilan entre mucho conocimiento (5) y ningún conocimiento (1) de: «el organigrama de mi organización», «los objetivos de mi organización o empresa» $\mathrm{y}$ «el convenio colectivo/estatuto de regulación».

13. Escala tipo Likert que oscila entre 1 (ninguna posibilidad de promoción) y 5 (muchas posibilidades) formada a partir de dos proposiciones: «con su formación, tiene posibilidades de que le asciendan en su empresa/organización» y «si continúo estudiando y formándome, puedo aspirar a un ascenso". 
Tabla 1. Relación entre sobreeducación (dos definiciones) y diversas variables ideacionales propias del estudio sociológico de las organizaciones (análisis de medias)

\begin{tabular}{|c|c|c|c|c|c|c|}
\hline \multirow[b]{3}{*}{ Variables dependientes } & \multicolumn{6}{|c|}{ Variables independientes } \\
\hline & \multicolumn{3}{|c|}{$\begin{array}{l}\text { Sobreeducación } 1 \text { : } \\
\text { universitarios según } \\
\text { los cinco estratos } \\
\text { ocupacionales }\end{array}$} & \multicolumn{3}{|c|}{$\begin{array}{l}\text { Sobreeducación 2: } \\
\text { estratos inferiores según } \\
\text { los cuatro niveles } \\
\text { educativos }\end{array}$} \\
\hline & $N$ & $F$ & $R^{2}$ & $N$ & $F$ & $R^{2}$ \\
\hline Satisfacción en el trabajo & 1.174 & $17,9^{* * *}$ & 0,054 & 2.318 & $4,4^{* * *}$ & 0,005 \\
\hline Interés por el propio puesto de trabajo & 1.184 & $57,6^{* * *}$ & 0,160 & 2.379 & 1,3 & 0,000 \\
\hline Interés por la empresa u organización & 1.168 & $32^{* * *}$ & 0,095 & 2.337 & $4,8^{* * *}$ & 0,005 \\
\hline Actitud ante el trabajo en general & 1.158 & $6,9^{* * *}$ & 0,018 & 2.326 & 0,5 & 0,001 \\
\hline Condiciones del puesto de trabajo & 1.187 & $13,7^{* * *}$ & 0,034 & 2.390 & 1,9 & 0,000 \\
\hline Alienación respecto al trabajo & 1.179 & $18^{* * *}$ & 0,049 & 2.370 & 0,5 & 0,000 \\
\hline Confianza con el entorno laboral & 1.179 & $5,8^{* * *}$ & 0,015 & 2.040 & $4,3^{* * *}$ & 0,005 \\
\hline Conocimiento de la organización & 991 & $11,8^{* * *}$ & 0,044 & 1.724 & $6,6^{* * *}$ & 0,010 \\
\hline Posibilidad de promoción & 954 & 1,2 & 0,002 & 1.700 & $27,9^{* * *}$ & 0,046 \\
\hline Cambio de empleo & 1.162 & $14,32^{* * *}$ & 0,046 & 2.288 & $9,86^{* * *}$ & ${ }^{k} 0,002$ \\
\hline
\end{tabular}

*** Significación menor o igual que 0,01 .

** Significación menor o igual que 0,05 pero mayor que 0,01 .

* Significación menor que 0,10 pero mayor que 0,05 .

Fuente: Encuesta de calidad de vida en el trabajo, 2003, elaboración propia.

rencia de niveles educativos (definición 2 de sobreeducación). Son los estratos y no el nivel de educación formal los que explican la desigualdad de resultados. Las actitudes ante la organización, los sentimientos hacia los compañeros o la empresa, la forma de entender el trabajo, etc. se explican más por el estrato de prestigio ocupacional que se ocupa que por el nivel educativo alcanzado. En resumen, es la ocupación y no el nivel educativo el que justifica en mayor medida la forma de pensar respecto al trabajo y a la empresa.

Desde el punto de vista del empresario o del organizador, tiene más importancia la definición segunda de sobreeducación. Las diferencias que hay entre contratar a alguien con exceso de educación para un puesto con escaso prestigio ocupacional o contratar a otra persona con el nivel educativo inferior para el mismo puesto, aparentemente más apropiado, no son muy grandes. Al fin y al cabo, lo que imprime carácter es el puesto de trabajo y no el nivel educativo, como ya se dijo. Contrate a quien contrate, el empresario u organizador se verá con una forma de pensar muy semejante hacia su trabajo y hacia la organización o empresa por parte del contratado.

No obstante, hay seis variables en las que parece influir el exceso de educación: la satisfacción en el trabajo, el interés por la empresa u organización, la confianza en el entorno laboral, el conocimiento de la organización, la posi- 
bilidad de promoción y el interés por cambiar de empleo. A partir de la información básica, más detallada, que no se ofrece por cuestión de espacio, sabemos que los sobreeducados, según la segunda definición, están menos satisfechos con su trabajo, tienen menos interés por la empresa u organización a la que prestan sus servicios, confían menos en el entorno laboral, tienen mayor conocimiento de la organización en la que trabajan (como era de esperar), piensan que tienen más posibilidades de promoción en la empresa u organización y declaran como muy posible el cambio de puesto de trabajo. Por supuesto, sabemos que hay muchos factores que influyen en estas variables relacionadas todas ellas con los aspectos laborales y la organización ${ }^{14}$.

Para controlar algunas variables externas, se ha realizado un análisis de regresión lineal en el que se considera como variables dependientes cinco variables arriba citadas (véase la tabla 2). Para controlar otras tres variables externas, se ha limitado la población analizada a los empleados (trabajadores por cuenta ajena) del sector privado con contrato laboral de carácter estable. Los resultados son evidentes, la sobreeducación del tipo segundo tiene que ver fundamentalmente con dos variables: con el conocimiento de la organización y con las expectativas de promoción del empleado. Los datos básicos indican, por su parte, que el conocimiento de la empresa aumenta cuanto menor sea la misma y cuanta mayor edad tenga el empleado, como era de suponer. Las expectativas de promoción es la otra variable estrechamente relacionada con la sobreeducación: cuanta mayor sea la sobreeducación, mayores expectativas de promoción (reales o supuestas) tendrá el empleado. Por lo demás, la sobreeducación, definida según el segundo criterio, no tiene que ver casi nada con las otras tres variables: la satisfacción en el trabajo, el interés por la empresa y la confianza en el entorno laboral.

Las variables que parecen explicar esa atonía organizacional por parte del empleado sobreducado son la "posibilidad de promoción" y el «interés por cambiar de empleo». Está claro que los sobreeducados tienen más expectativas de promoción que el resto de la población que ocupa los estratos ocupacionales inferiores. Estas expectativas forman parte de la aceptación del proceso en el que el empleado sobreeducado se encuentra inmerso. Para corroborar esta hipótesis, se ha realizado el análisis de regresión de la tabla 3.

En la tabla 3 se muestran los resultados de un segundo modelo de regresión lineal en el que se ha añadido la variable "posibilidades de promoción» a las variables independientes anteriores, las del modelo 1 de regresión. Se aprecia entonces que el modelo aumenta considerablemente la proporción de la varianza explicada de las dos variables dependientes. Pero lo que más llama la atención es que, una vez controlada la variable "posibilidades de promoción», la población sobreeducada presenta insatisfacción en el trabajo y aparenta desinterés por la empresa.

14. Parece ser que la formación en el puesto de trabajo es un factor que facilita también la movilidad de los sobreeducados. En este trabajo, no se contempla tal variable (véase M. PollmannSchult y F. Buchel, 2004; R. Dekker, A. Grip y H. Heijke, 2002; A. Chevalier, 2003). 
Tabla 2. Modelo 1 de regresión lineal de varias variables dependientes ideacionales de tipo organizacional. Población formada por empleados del sector privado con contrato laboral estable

Variables dependientes: coeficientes Beta estandarizados

\begin{tabular}{llllll}
$\begin{array}{l}\text { Variables } \\
\text { independientes }\end{array}$ & $\begin{array}{l}\text { Satisfacción } \\
\text { en el trabajo }\end{array}$ & $\begin{array}{l}\text { Posibilidades } \\
\text { de promoción }\end{array}$ & $\begin{array}{l}\text { Interés por la } \\
\text { empresa }\end{array}$ & $\begin{array}{l}\text { Confianza en el } \\
\text { entorno laboral }\end{array}$ & $\begin{array}{l}\text { Conocimiento } \\
\text { de la organización }\end{array}$ \\
\hline $\begin{array}{l}\text { Tamaño de } \\
\text { la empresa }\end{array}$ & $\begin{array}{l}0,012 \\
(0,346)\end{array}$ & $\begin{array}{l}0,029 \\
(0,786)\end{array}$ & $\begin{array}{l}-0,026 \\
(-0,775)\end{array}$ & $\begin{array}{l}-0,039 \\
(-1,151)\end{array}$ & $\begin{array}{l}-0,112 \\
(3,312)^{* * *}\end{array}$ \\
\hline $\begin{array}{l}\text { Ingresos } \\
\text { del entrevistado }\end{array}$ & 0,020 & 0,255 & 0,026 & 0,046 & $-0,054$ \\
\hline Edad & $(0,597)$ & $(6,828)^{* * *}$ & $(0,778)$ & $(1,370)$ & $(-1,589)$ \\
del entrevistado & $(1,433)$ & $(-5,88)^{* * *}$ & $(2,420)^{* *}$ & $(0,124)$ & 0,106 \\
\hline Sobreeducación 2 & $-0,054$ & 0,165 & 0,016 & $-0,016$ & 0,164 \\
\hline Constante & $(-1,545)$ & $(4,337)^{* * *}$ & $(0,457)$ & $(-0,458)$ & $(4,748)^{* * *}$ \\
\hline$N$ & $(20,171)^{* * *}$ & $(5,881)^{* * *}$ & $(22,401)^{* * *}$ & $(26,073)^{* * *}$ & $(12,243)^{* * *}$ \\
\hline$R$-cuadrado & 0,008 & 0,166 & 0,008 & 0,004 & 853 \\
\hline$F$ & 1,696 & $32,46^{* * *}$ & 1,831 & 0,794 & 0,047 \\
\hline
\end{tabular}

Significación menor o igual que 0,01 .

** Significación menor o igual que 0,05 pero mayor que 0,01 .

* Significación menor que 0,10 pero mayor que 0,05 .

Entre paréntesis, el estadístico $t$ de STUDENT.

Fuente: Encuesta de calidad de vida en el trabajo, 2003, elaboración propia.

Además, la población sobreeducada (definición segunda) también se declara mucho más interesada en cambiar de puesto de trabajo ${ }^{15}$. Sin embargo, en este caso la relación no es lineal. Solamente el caso más extremo, el de los universitarios que ocupan posiciones laborales inferiores, es el que admite claramente tal posibilidad. De cualquier forma, el admitir tal posibilidad de cambio tiene que influir en el atemperamiento de la insatisfacción inmediata.

Hay que destacar también que la sobreeducación apenas tiene que ver con el pluriempleo (que, en general, es muy bajo en las cuatro categorías estudiadas). Así mismo, tampoco tiene que ver especialmente con la tendencia a sindicarse.

De acuerdo con todo lo anterior, el empleador no gana ni pierde mucho contratando a personas sobreeducadas (según esta segunda definición), siempre que estén abiertas las expectativas de promoción por parte de los empleados sobreeducados, ya sea en la misma empresa o en otra. Esto se relaciona, a su vez, con la temporalidad o la continuidad de la situación de sobreeducación que aquí no se ha tratado (véase S. Rubb, 2003).

15. Este resultado coincide con el de S. Wald (2005), según el cual, la población sobreeducada es mucho más activa a la hora de buscar empleo. 
Tabla 3. Modelo 2 de regresión lineal de diversas variables dependientes ideacionales de tipo organizacional. Población formada por empleados del sector privado con contrato laboral estable

\begin{tabular}{lll}
\hline \multirow{2}{*}{$\begin{array}{l}\text { Variables } \\
\text { independientes }\end{array}$} & $\begin{array}{l}\text { Variables dependientes: } \\
\text { coeficientes Beta estandarizados }\end{array}$ \\
\cline { 2 - 3 } Satisfacción en el trabajo & Interés por la empresa \\
\hline Posibilidades de promoción & 0,114 & 0,07 \\
& $(2,704)^{* * *}$ & $(1,744)^{*}$ \\
\hline Tamaño de la empresa & $-0,087$ & $-0,07$ \\
& $(-2,166)^{* *}$ & $(-1,848)^{*}$ \\
\hline Edad del entrevistado & 0,064 & 0,132 \\
& $(1,518)$ & $(3,283)^{* * *}$ \\
\hline Ingresos del entrevistado & 0,177 & 0,291 \\
& $(4,241)^{* * *}$ & $(7,338)^{* * *}$ \\
\hline Sobreeducación 2 & $-0,109$ & $-0,131$ \\
& $(-2,639)^{* * *}$ & $(-3,312)^{* * *}$ \\
\hline Constante & $(13,71)^{* * *}$ & $(17,182)^{* * *}$ \\
\hline$N$ & 638 & 651 \\
\hline$R$-cuadrado & 0,062 & 0,129 \\
\hline$F$ & $8,329^{* * *}$ & $19,093^{* * *}$ \\
\hline
\end{tabular}

*** Significación menor o igual que 0,01 .

** Significación menor o igual que 0,05 pero mayor que 0,01 .

* Significación menor que 0,10 pero mayor que 0,05 .

Entre paréntesis, el estadístico $t$ de STUDENT.

Fuente: Encuesta de calidad de vida en el trabajo, 2003, elaboración propia.

\section{Consecuencias privadas de la sobreeducación}

Se entiende aquí como consecuencias privadas las diversas declaraciones sobre satisfacción ${ }^{16}$ y grado de felicidad ${ }^{17}$ planteadas en el cuestionario de la Encuesta de calidad de vida en el trabajo. Son las siguientes:

1. Satisfacción en el trabajo (también usado en el análisis anterior).

2. Satisfacción con las condiciones materiales de la casa en que vive.

3. Satisfacción con la situación económica del hogar.

4. Satisfacción con el tiempo libre de que dispone.

5. Satisfacción con la vida que tiene en la actualidad.

16. Todas las declaraciones sobre satisfacción se efectúan en una escala tipo Cantril, en la que (1) es muy insatisfecho y (10) es muy satisfecho.

17. La pregunta sobre el grado de felicidad admite cuatro respuestas: (1) muy feliz; (2) bastante feliz; (3) no muy feliz, y (4) nada feliz. 
6. Satisfacción global (media de las cuatro anteriores — no entra en la media la satisfacción en el trabajo).

7. Grado de felicidad.

Los resultados del análisis de medias se exponen en la tabla 4. Casi todas las variables dependientes tienen que ver con los dos tipos de sobreeducación. Solamente la variable "grado de felicidad» se relaciona algo menos con la sobreeducación definida según el primer criterio. Utilizando de nuevo los datos básicos, que aquí no se exponen, sabemos que (según la primera definición de sobreeducación) los universitarios que pertenecen a un estrato más alto están más satisfechos y son más felices que los de los estratos inferiores, como era de esperar. También sabemos que, según la segunda definición, los que ocupan los estratos inferiores que tienen estudios superiores están menos satisfechos que los que tienen niveles educativos formales inferiores. En general, los más sobreeducados (según esta segunda definición) presentan niveles de satisfacción por debajo de la media de su categoría de estrato ocupacional, excepto en la satisfacción con la casa y la situación económica del hogar, cuyos resultados se acercan a la media. No obstante, están por encima de la media en el grado de felicidad declarado. La población sobreeducada, en este caso, se declara más feliz que el resto.

Tabla 4. Relación entre sobreeducación (dos definiciones), felicidad y diversas formas de entender la satisfacción (análisis de medias)

\begin{tabular}{|c|c|c|c|c|c|c|}
\hline \multirow[b]{3}{*}{ Variables dependientes } & \multicolumn{6}{|c|}{ Variables independientes } \\
\hline & \multicolumn{3}{|c|}{$\begin{array}{l}\text { Sobreeducación 1: } \\
\text { universitarios según } \\
\text { los cinco estratos } \\
\text { ocupacionales }\end{array}$} & \multicolumn{3}{|c|}{$\begin{array}{l}\text { Sobreeducación 2: } \\
\text { estratos inferiores según } \\
\text { los cuatro niveles } \\
\text { educativos }\end{array}$} \\
\hline & $N$ & $F$ & $R^{2}$ & $N$ & $F$ & $R^{2}$ \\
\hline Satisfacción en el trabajo & 1.174 & $17,9^{* * *}$ & 0,054 & 2.318 & $4,4^{* * *}$ & 0,005 \\
\hline Satisfacción con el salario & 1.182 & $14,55^{* * *}$ & 0,045 & 2.374 & $3,59^{* *}$ & 0,0 \\
\hline $\begin{array}{l}\text { Satisfacción con la situación } \\
\text { económica del hogar }\end{array}$ & 1.179 & $12,1^{* * *}$ & 0,039 & 2.379 & $11,8^{* * *}$ & 0,01 \\
\hline Satisfacción con el tiempo libre & 1.184 & $4,8^{* * *}$ & 0,013 & 2.383 & $4,4^{* * *}$ & 0,001 \\
\hline Satisfacción con la vida que tiene & 1.181 & $7,1^{* * *}$ & 0,023 & 2.379 & $4,6^{* * *}$ & 0,001 \\
\hline Satisfacción global & 1.172 & $12,2^{* * *}$ & 0,039 & 2.360 & $7,04^{* * *}$ & 0,003 \\
\hline Grado de felicidad & 1.165 & 1,4 & 0,070 & 2.356 & $4,7^{* * *}$ & 0,078 \\
\hline
\end{tabular}

Significación menor o igual que 0,01 .

** Significación menor o igual que 0,05 pero mayor que 0,01 .

* Significación menor que 0,10 pero mayor que 0,05 .

Fuente: Encuesta de calidad de vida en el trabajo, 2003, elaboración propia. 
Sin embargo, la relación causal aparente anterior desaparece cuando se controla con las variables «tamaño del hábitat», «edad» e «ingresos». Los resultados de tal control se aprecian en el análisis de regresión de la tabla 5. De acuerdo con ellos, no parece que haya una relación evidente entre la sobreeducación (de acuerdo con la segunda definición) y las manifestaciones de satisfacción y felicidad.

El interés por dedicar más o menos tiempo a un listado de actividades no laborales es uno de los mecanismos de compensación de la sensación negativa de sobreeducación. Formaría parte del proceso de privatización de ese sentimiento. Las variables de este tipo que se van a tener en cuenta ahora son las siguientes:

1. Interés por dedicar tiempo al trabajo (los valores oscilan desde 1, quiere dedicar mucho menos tiempo; hasta 5 , quiere dedicar mucho más tiempo).

2. Interés por dedicar tiempo a las labores de casa (los mismos valores).

3. Interés por estar con la familia (los mismos valores).

4. Interés por estar con los amigos (los mismos valores).

Tabla 5. Modelo de regresión de varias variables dependientes ideacionales de tipo personal. Población formada por empleados del sector privado con contrato laboral estable

\begin{tabular}{lll}
\hline \multirow{2}{*}{$\begin{array}{l}\text { Variables } \\
\text { independientes }\end{array}$} & \multicolumn{2}{l}{$\begin{array}{l}\text { Variables dependientes: } \\
\text { coeficientes Beta estandarizados }\end{array}$} \\
\cline { 2 - 3 } Satisfacción global & Grado de felicidad \\
\hline Tamaño del hábitat & $\begin{array}{l}-0,118 \\
(-3,277)^{* * *}\end{array}$ & $\begin{array}{l}0,075 \\
(2,066)^{* *}\end{array}$ \\
\hline Edad del entrevistado & $-0,008$ & 0,049 \\
& $(-0,223)$ & $(1,292)$ \\
\hline Ingresos del entrevistado & 0,118 & $-0,88$ \\
& $(3,245)^{* * *}$ & $(-2,396)^{* *}$ \\
\hline Sobreeducación 2 & $-0,019$ & $-0,018$ \\
& $(-0,500)$ & $(-0,474)$ \\
\hline Constante & $(24,905)^{* * *}$ & $(17,183)^{* * *}$ \\
\hline$N$ & 765 & 767 \\
\hline R-cuadrado & 0,030 & 0,017 \\
\hline$F$ & $5,792^{* * *}$ & $3,227^{* *}$ \\
\hline
\end{tabular}

*** Significación menor o igual que 0,01 .

** Significación menor o igual que 0,05 pero mayor que 0,01 .

* Significación menor que 0,10 pero mayor que 0,05 .

Entre paréntesis, el estadístico $t$ de STUDENT.

Fuente: Encuesta de calidad de vida en el trabajo, 2003, elaboración propia. 
5. Interés por actividades de ocio y aficiones (los mismos valores).

6. Interés por descansar (los mismos valores).

7. Interés por tiempo libre (media de las variables 2, 3, 4 y 5 anteriores. Los valores oscilan entre 1, quiere dedicar menos tiempo al tiempo libre, y 10, quiere dedicar más tiempo al tiempo libre).

Según los datos de la tabla 6, la sobreeducación 2 tiene mucho que ver con el interés por dedicar más o menos tiempo a los amigos, al ocio y a las aficiones. También tiene que ver, aunque menos, con el interés por dedicar más o menos tiempo a las tareas domésticas y a descansar. Si entramos en detalle, volvemos a los datos básicos, descubrimos que la población, cuanto más sobreeducada está, más interés tiene por dedicar tiempo a los amigos, al ocio y a las aficiones, y menos por descansar. El interés por descansar está más relacionado con los grados inferiores de sobreeducación (niveles inferiores de educación de las ocupaciones menos prestigiadas). Así pues, el índice global («interés por el tiempo libre») se ve distorsionado por esa diferencia de comportamiento de tres variables, de las cuatro, que lo conforman: interés por los amigos, las aficiones y el descanso.

Tabla 6. Relación entre sobreeducación (dos definiciones) e interés por diversas formas de dedicación del tiempo (análisis de medias)

\begin{tabular}{|c|c|c|c|c|c|c|}
\hline \multirow{3}{*}{$\begin{array}{l}\text { Variables dependientes } \\
\text { Interés por dedicar más tiempo }\end{array}$} & \multicolumn{6}{|c|}{ Variables independientes } \\
\hline & \multicolumn{3}{|c|}{$\begin{array}{l}\text { Sobreeducación 1: } \\
\text { universitarios según } \\
\text { los cinco estratos } \\
\text { ocupacionales }\end{array}$} & \multicolumn{3}{|c|}{$\begin{array}{l}\text { Sobreeducación } 2 \text { : } \\
\text { estratos inferiores según } \\
\text { los cuatro niveles } \\
\text { educativos }\end{array}$} \\
\hline & $N$ & $F$ & $R^{2}$ & $N$ & $F$ & $R^{2}$ \\
\hline Al trabajo & 1.162 & $4,966^{* * *}$ & 0,011 & 2.303 & 0,988 & 0,000 \\
\hline A labores de casa & 1.170 & 0.614 & 0,001 & 2.335 & $2,306^{*}$ & 0,002 \\
\hline A estar con la familia & 1.174 & $2,026^{*}$ & 0,002 & 2.346 & 1,718 & 0,001 \\
\hline A estar con los amigos & 1.173 & 0,877 & 0,001 & 2.334 & $9,679^{* * *}$ & 0,009 \\
\hline A ocio y aficiones & 1.173 & 0,656 & 0,001 & 2.333 & $5,712^{* * *}$ & 0,006 \\
\hline A descansar & 1.172 & $2,157^{*}$ & 0,005 & 2.340 & $2,34^{*}$ & 0,002 \\
\hline A tiempo libre (índice) & 1.165 & 1,416 & 0,003 & 2.318 & $2,784^{* *}$ & 0,002 \\
\hline $\mathrm{Al}$ asociacionismo (índice) & 1.145 & $9,22^{* * *}$ & 0,027 & 2.347 & $6,63^{* * *}$ & 0,007 \\
\hline
\end{tabular}

*** Significación menor o igual que 0,01 .

** Significación menor o igual que 0,05 pero mayor que 0,01 .

* Significación menor que 0,10 pero mayor que 0,05 .

Fuente: Encuesta de calidad de vida en el trabajo, 2003, elaboración propia. 
Tabla 7. Modelo de regresión de tres variables dependientes sobre utilización del tiempo

\begin{tabular}{lllll}
\hline \multirow{2}{*}{$\begin{array}{l}\text { Variables } \\
\text { independientes }\end{array}$} & \begin{tabular}{l} 
Variables dependientes: coeficientes Beta estandarizados \\
\cline { 2 - 5 } los amigos
\end{tabular} & $\begin{array}{l}\text { A ocio } \\
\text { y aficiones }\end{array}$ & $\begin{array}{l}\text { Tiempo } \\
\text { libre }\end{array}$ & $\begin{array}{l}\text { Índice de } \\
\text { asociacionismo }\end{array}$ \\
\hline Tamaño del hábitat & 0,018 & $-0,012$ & 0,004 & 0,032 \\
& $(0,777)$ & $(-0,532)$ & $(0,169)$ & $(1,436)$ \\
\hline Edad del entrevistado & $-0,156$ & $-0,114$ & $-0,069$ & $-0,064$ \\
& $(-6,607)^{* * *}$ & $(-0,4,798)^{* * *}$ & $(-2,908)^{* * *}$ & $(-2,684)^{* * *}$ \\
\hline Ingresos del entrevistado & 0,004 & 0,075 & 0,072 & $-0,088$ \\
& $(0,186)$ & $(3,286)^{* * *}$ & $(3,123)^{* * *}$ & $(-3,856)^{* * *}$ \\
\hline Sobreeducación 2 & 0,052 & 0,044 & 0,023 & $-0,093$ \\
& $(2,22)^{* *}$ & $(1,853)^{*}$ & $(0,981)$ & $(-3,924)^{* * *}$ \\
\hline Constante & $(40,942)^{* * *}$ & $(39,232)^{* * *}$ & $(50,209)^{* * *}$ & $(145,958)^{* * *}$ \\
\hline$N$ & 1.924 & 1.923 & 1.911 & 1.938 \\
\hline$R$-cuadrado & 0,032 & 0,021 & 0,010 & 0,021 \\
\hline$F$ & $15,681^{* * *}$ & $10,38^{* * *}$ & $4,977^{* * *}$ & $10,514^{* * *}$ \\
\hline
\end{tabular}

Significación menor o igual que 0,01 .

** Significación menor o igual que 0,05 pero mayor que 0,01 .

* Significación menor que 0,10 pero mayor que 0,05 .

Entre paréntesis, el estadístico $t$ de STUDENT.

Fuente: Encuesta de calidad de vida en el trabajo, 2003, elaboración propia.

Según los datos del análisis de regresión lineal de la tabla $7^{18}$, la edad y los ingresos explican mejor que la sobreeducación el interés por dedicar tiempo a los amigos, las aficiones y, en general, al tiempo libre. No obstante, la dedicación a los amigos parece que tiene más que ver con la sobreeducación que con los ingresos. De cualquier forma y en resumen, la dedicación de tiempo a otros intereses no laborales (en este caso, a los amigos, al ocio y a las aficiones) está relacionada claramente con la sobreeducación 2. Los amigos y las aficiones pueden constituir una válvula de escape a la insatisfacción posible generada por la sobreeducación.

Otra forma de privatizar la insatisfacción es formar parte de asociaciones en las que, presumiblemente, se ocupan posiciones más acordes con el nivel educativo. Se trata de otro mecanismo de compensación. La Encuesta de calidad de vida en el trabajo inquiere sobre la pertenencia a nueve tipos de orga-

18. El análisis de regresión lineal que sigue (tabla 7) se ha efectuado sobre toda la población de los estratos ocupacionales inferiores (a diferencia de los modelos de regresión anteriores), porque la proporción de varianza explicada es más alto que si nos hubiéramos ceñido a la población empleada en el sector privado con contrato estable. 
nización (el noveno es residual: «otros»). La respuesta presenta tres posibilidades: 1) pertenece como miembro activo; 2) pertenece como miembro no activo, y 3) no pertenece a ninguna asociación de ese tipo. Para facilitar su análisis, se ha elaborado un "índice de asociacionismo" que oscila entre 3 (muy alto) y 9 (nada). El asociacionismo está muy relacionado con ambas definiciones de sobreeducación, tal y como se desprende de la tabla 6 . En líneas generales y manejando los datos básicos, cuanta mayor sea la sobreeducación, mayor es el interés por pertenecer a asociaciones. El análisis de regresión de la tabla 7 indica que la sobreeducación, en su segunda definición, tiene que ver claramente con el índice de asociacionismo, al igual que los ingresos y la edad.

Toda la población en cuestión tiene poco interés por las asociaciones. No obstante, dentro de esa tónica general, los universitarios que ocupan posiciones laborales poco prestigiadas (sobreeducación 2) se apuntan más a organizaciones vecinales, culturales, benéficas, profesionales y políticas. Vaya por delante que la pertenencia a partidos políticos es mínima en todas las categorías. Así mismo, esos universitarios tienen poco interés por las asociaciones deportivas y las religiosas.

\section{Consecuencias sociales y políticas de la sobreeducación}

Se recoge, a continuación, un conjunto de variables dependientes cuya posible influencia va más allá de la vida privada y laboral del entrevistado. Tales variables pueden interpretarse como indicadores de un comportamiento político mucho más amplio que su propia apariencia. Estas variables son cinco:

1. Identificación de clase (desde 1 , clase más baja; hasta 10 , clase más alta $)^{19}$.

2. Radicalismo político (desde 1, muy radical; hasta 3, nada radical) ${ }^{20}$.

3. Izquierda / derecha (desde 1, extrema izquierda; hasta 7, extrema derecha) ${ }^{21}$.

4. Religiosidad (desde 1, nada practicante; hasta 10, muy practicante ${ }^{22}$.

5. Interés por la política (desde 1, muy interesado; hasta 4, nada interesado $)^{23}$.

Las cinco variables están relacionadas con la sobreeducación del tipo 2, en mayor o menor medida. No ocurre lo mismo con la sobreeducación del tipo 1. En este caso, ni la ideología derecha / izquierda ni la religiosidad tienen nada que ver con la sobreeducación.

19. Escala tipo Cantril.

20. Escala tipo Likert formada por tres niveles de aceptación: 1, la ha hecho; 2, podría hacerla, y 3, nunca la haría. Aplicada a cuatro proposiciones: $a$ ) firmar una petición; $b$ ) secundar boicots; $c$ ) participar en huelgas, y $d$ ) ocupar edificios o fábricas.

21. Escala tipo Cantril. Los datos de esta escala, así como los demás del análisis correspondiente, son de la encuesta de 1999, porque la pregunta no se repite en la del 2003.

22. Escala tipo Cantril. Los datos de esta escala, así como los demás del análisis correspondiente, son de la encuesta de 1999, porque la pregunta no se repite en la del 2003.

23. Escala tipo Cantril. 
Tabla 8. Relación entre sobreeducación (dos definiciones) y diversas variables ideacionales

\begin{tabular}{|c|c|c|c|c|c|c|}
\hline \multirow[b]{3}{*}{ Variables dependientes } & \multicolumn{6}{|c|}{ Variables independientes } \\
\hline & \multicolumn{3}{|c|}{$\begin{array}{l}\text { Sobreeducación 1: } \\
\text { universitarios según } \\
\text { los cinco estratos } \\
\text { ocupacionales }\end{array}$} & \multicolumn{3}{|c|}{$\begin{array}{l}\text { Sobreeducación 2: } \\
\text { estratos inferiores según } \\
\text { los cuatro niveles } \\
\text { educativos }\end{array}$} \\
\hline & $N$ & $F$ & $R^{2}$ & $N$ & $F$ & $R^{2}$ \\
\hline Identificación de clase & 1.145 & $9,22^{* * *}$ & 0,027 & 2.347 & $6,625^{* * *}$ & 0,007 \\
\hline Radicalismo político & 1.113 & $2,453^{* *}$ & 0,007 & 2.207 & $31,623^{* * *}$ & 0,036 \\
\hline Izquierda / derecha (1999) & 843 & 0,558 & 0,001 & 974 & $2,42^{*}$ & 0,0 \\
\hline Religiosidad (1999) & 843 & 1,092 & 0,0 & 1.628 & $3,61^{* *}$ & 0,0 \\
\hline Interés por la política & 1.170 & $3,844^{* * *}$ & 0,04 & 2.353 & $41,62^{* * *}$ & 0,049 \\
\hline
\end{tabular}

** Significación menor o igual que 0,01 .

** Significación menor o igual que 0,05 pero mayor que 0,01 .

* Significación menor que 0,10 pero mayor que 0,05 .

Fuente: Encuesta de calidad de vida en el trabajo, 1999 y 2003, elaboración propia.

Observando los datos básicos más detallados que no aparecen en el texto, se aprecia que, cuanto mas alto es el estrato ocupacional de los universitarios, más alta es también la clase con la que se identifican, como era de suponer. Algo parecido ocurre con los estudios: cuanto más alto es el nivel educativo, más alta es la clase con la que se identifican, aunque en este último caso los datos no son tan acusados. Los datos sobre el radicalismo político no parecen encajar con lo esperado: cuanto más alto es el estrato ocupacional de los universitarios (sobreeducación 1), mayor es también el radicalismo político. Esto todavía se acentúa más con la sobreeducación del segundo tipo: cuanto más alto es el nivel educativo de los estratos ocupacionales inferiores, mayor también es el radicalismo político. Ésta es una de las pocas veces en que la desigualdad de estudios formales (sobreeducación 2) tiene más influencia sobre la variable dependiente que la desigualdad de estratos (sobreeducación 1).

Por su parte, los universitarios pertenecientes a los estratos ocupacionales inferiores (sobreeducación 2) se declaran más de derechas que el resto. No existe, sin embargo, una relación lineal: los más izquierdosos, en este caso, son los que poseen niveles medios educativos. Así mismo, los universitarios de esa categoría ocupacional también evidencian una mayor práctica religiosa. En ambos casos, las diferencias con las demás categorías son escasas, pese a lo cual se puede afirmar que la sobreeducación (tipo 2) favorece la ideología de derechas y la práctica religiosa.

El interés por la política depende más del nivel educativo que del estrato ocupacional. En general, cuanto más alto sea el nivel educativo (sobreeducación 2), mayor será el interés por la política. Sin embargo, no es tan clara la 
Tabla 9. Modelo de regresión de cuatro variables dependientes de tipo social y político

\begin{tabular}{lllll}
\hline \multirow{2}{*}{$\begin{array}{l}\text { Variables } \\
\text { independientes }\end{array}$} & \begin{tabular}{l} 
Variables dependientes: coeficientes Beta estandarizados \\
\cline { 2 - 5 } de clase
\end{tabular} & $\begin{array}{l}\text { Radicalismo } \\
\text { político }\end{array}$ & $\begin{array}{l}\text { Religiosidad } \\
(1999)\end{array}$ & $\begin{array}{l}\text { Interés } \\
\text { por la política }\end{array}$ \\
\hline Tamaño del hábitat & 0,0 & 0,003 & $-0,011$ & $-0,019$ \\
& $(-0,02)$ & $(0,111)$ & $(0,411)$ & $(-0,87)$ \\
\hline Edad del entrevistado & $-0,06$ & 0,076 & 0,179 & $-0,076$ \\
& $(-2,542)^{* *}$ & $(3,188)^{* * *}$ & $(6,18)^{* * *}$ & $(-3,279)^{* * *}$ \\
\hline Ingresos del entrevistado & 0,178 & $-0,102$ & $-0,12$ & $-0,108$ \\
& $(7,811)^{* * *}$ & $(-4,455)^{* * *}$ & $\left(-4,348^{* * *}\right.$ & $(-4,809)^{* * *}$ \\
\hline Sobreeducación 2 & 0,098 & $-0,178$ & 0,072 & $-0,223$ \\
& $(4,188)^{* * *}$ & $(-7,531)^{* * *}$ & $(2,478)^{* *}$ & $(-9,376)^{* * *}$ \\
\hline Constante & $(33,553)^{* * *}$ & $(40,647)^{* * *}$ & $(7,272)^{* * *}$ & $41,589^{* * *}$ \\
\hline$N$ & 1.912 & 1.855 & 1.295 & 1.940 \\
\hline$R$-cuadrado & 0,048 & 0,056 & 0,038 & 0,065 \\
\hline$F$ & $23,86^{* * *}$ & $27,331^{* * *}$ & $12,89^{* * *}$ & $33,621^{* * *}$ \\
\hline
\end{tabular}

*** Significación menor o igual que 0,01 .

** Significación menor o igual que 0,05 pero mayor que 0,01 .

* Significación menor que 0,10 pero mayor que 0,05 .

Entre paréntesis, el estadístico $t$ de STUDENT.

Fuente: Encuesta de calidad de vida en el trabajo, 1999 y 2003, elaboración propia.

relación con los estratos (sobreeducación 1): entre los universitarios, el estrato más interesado por la política es el medio bajo y luego el alto.

El análisis de regresión (tabla 9) nos indica que, una vez controladas las variables "tamaño del hábitat», "edad» e «ingresos», la sobreeducación según su segunda definición tiene claramente que ver con las cuatro variables dependientes: identificación de clase, radicalismo politico, religiosidad e interés por la politica. Se puede afirmar, por lo tanto, que la población sobreeducada (segundo criterio) prefiere trasladar su insatisfacción al ámbito público. En otras palabras, a diferencia de la población norteamericana, que "privatiza el descontento" (V. Burris, 1983), la población correspondiente en España proyecta tal descontento en el espacio público. Seguramente, mediante un proceso mental de culpabilizar al estado o a la sociedad. Indicadores evidentes de tal situación son el mayor interés por la política y el mayor nivel de radicalismo político entre los sobreeducados (tipo 2).

\section{Conclusiones}

Nuestro objetivo ha sido estudiar los efectos de la sobreeducación en la sociedad española actual. La educación es un factor relativamente controlado por el actor que sirve para mantener o mejorar el estatus. Por lo tanto, se debe entender que la sobreeducación es un fracaso y produce algún tipo de desazón que 
se manifiesta y se compensa de maneras diversas. De acuerdo con esto, se han determinado tres ámbitos en los que puede tener lugar esa desazón y su compensación posible: el ámbito laboral, el privado y el político.

Pero también, en una sociedad más moderna, se produce un mayor grado de incoherencia social y cultural en las mismas personas que en una sociedad más tradicional. En una sociedad moderna, se acaba por aceptar más fácilmente como normal la incoherencia entre educación y empleo. Posiblemente esto explique las escasas consecuencias sociales de la sobreeducación.

Para observar las consecuencias en cuestión, se ha definido operativamente la sobreeducación como un continuo que oscila entre 1 , nada sobreeducada (población ocupada en empleos de prestigio medio-bajo y bajo, sin nivel de estudios), y 4, muy sobreeducada (población de los mismos estratos ocupacionales bajos, con nivel de estudios universitario). De esta forma, se ha obtenido una variable continua de cuatro valores (sin estudios, con estudios primarios, medios y superiores). Como es lógico, no forma parte de esta definición el total de la población encuestada, sólo una parte de la misma: la que pertenece a los dos estratos inferiores de la jerarquía ocupacional. Es una variable que sirve únicamente para establecer relaciones explicativas entre variables, no sirve para describir la situación de sobreeducación de una población cualquiera.

Por su parte, las consecuencias posibles han sido clasificadas en tres categorías: consecuencias empresariales u organizacionales, consecuencias privadas y consecuencias de carácter social o político. Se supone que los problemas de sobreeducación en la empresa u organización han de ser solucionados por el empresario o empleador, los problemas privados han de ser solucionados por cada cual y los públicos han de ser solucionados por los políticos correspondientes.

La sobreeducación no plantea problemas en la empresa. La población con estas características no es especialmente conflictiva ni está especialmente en contra del funcionamiento de la empresa u organización. Sin embargo, para que esa situación se mantenga, parece importante que la persona sobreeducada debe pensar que tiene expectativas de promoción, bien dentro de la empresa o bien en otra (el interés por cambiar de puesto de trabajo entre los sobreeducados es muy alto). En caso contrario, la población sobreeducada presenta insatisfacción con el trabajo y escaso interés por la empresa.

No parece tampoco que la sobreeducación afecte mucho a la vida privada de la población en cuestión. Los niveles de satisfacción son semejantes a los del resto de la población. Esto se corresponde, no obstante, con un mayor interés por parte de los sobreeducados por estar con los amigos y por llevar a cabo actividades de ocio y aficiones. Se aprecia que los amigos y las aficiones pueden servir de compensación a nivel privado. Asimismo, la población sobreeducada tiene bastante más propensión a pertenecer a asociaciones (sobre todo asociaciones vecinales, culturales, benéficas, profesionales y políticas). En cierta forma, esta propensión al asociacionismo puede considerarse como otra forma de compensación privada al presumible descontento de la sobreeducación. 
Donde sí hay diferencias evidentes es con las variables que se han denominado sociales y políticas. Especialmente en el interés por la política. En España, el interés por la política es muy bajo en general, sin embargo, la población sobreeducada tiene un marcado interés por hablar de política con su entorno social. También está más radicalizada, está más dispuesta a llevar a cabo actividades violentas para hacer valer sus derechos. No obstante, es ligeramente más de derechas y levemente más religiosa que el resto de la población no sobreeducada. Estas últimas tendencias (la ideología levemente más de derechas y cierta mayor religiosidad) tienen mucho que ver con el último rasgo interesante del análisis: la población sobreeducada tiende a identificarse con una clase social más alta que la no sobreeducada.

Se puede concluir, a la vista de lo anterior, que la población sobreeducada en España prefiere volcar al espacio público su descontento o insatisfacción, en vez de hacerlo contra la empresa u organización en la que trabaja o contra sí mismo. Este tipo de persona consigue compensar de una u otra forma su desazón sin proyectarla sobre el ámbito laboral ni sobre el privado. Posiblemente culpen de su situación más al estado y a la sociedad que a otra cosa.

\section{Bibliografía}

Battu, H.; Belfield, C. R.; Sloane, P. J. (2000). «Over-education: How sensitive are the measures?». National Institute Economic Review, 171, p. 82-93.

BÉDUWÉ, C.; PlanAS, J. Expansión educativa y mercado de trabajo. Estudio comparativo realizado en cinco países europeos: Alemania, España, Francia, Italia, Reino Unido, con referencia a los Estados Unidos [en línea]. Instituto Nacional de las Cualificaciones. $<$ http://edex.univ-tlse1.fr/edex/>.

BERG, I. (1970). Education and Jobs: The Great Training Robbery. Nueva York: Praeger. Bouchel, F. (2002). "The effects of overeducation on productivity in Germany: The firms' viewpoint». Economics of Education Review, 21, p. 263-275.

Buchel, F.; Mertens, A. (2.004). "Overeducation, undereducation, and the theory of career mobility». Applied Economics, 36, p. 803-316.

Buchel, F.; De Grip, A.; Mertens, A. (2003). Overeducation in Europe. Cheltenham (UK): Edward Elgar.

BurRIS, B.-H. (1983). «The human effects of underemployment». Social Problems. Kalamazoo, Mich, 31, p. 96-110.

BuRRIS, V. (1983). «The social and political consequences of overeducation». American Sociological Review, 48, p. 454-467.

CARABAÑA, J.; BuenO, C. (1996). Escalas de prestigio ocupacional. Madrid: CIS.

Charlot, O.; Decreuse, B. (2005). «Self-selection in education with matching frictions». Labour Economics, 12, p. 251-267.

Chevalier, A. (2003). «Measuring over-education». Económica, 70, p. 509-531.

DeKKER, R.; GRIP, A.; HeIJKE, H. (2002). "The effects of training and overeducation on career mobility in a segmented labour market». International Journal of Manpower, 23, p. 106-125.

Folger, J.; NAM, Ch.B. (1964). «Trends in education in relation to the occupational structure». Sociology of Education, 38, p. 19-33. 
GARMENDiA, J. A. (2004). «Impacto de la cultura en los resultados de la organización». Revista Española de Investigaciones Sociológicas, 108, p. 75-96.

Gobernado, R. (1996a). "Los límites de la expansión educativa». Revista Española de Pedagogía, 205, p. 487-510.

- (1996b). "El logro ocupacional en España, veinte años después». Revista Española de Investigaciones Sociológicas», 74, p. 209-224.

- (2003). «El valor de la educación en el empleo del sector privado». Papers, 69, p. 11-29.

- (2005). La sobreeducación en España: estudio descriptivo y revisión critica del concepto [en línea]. <www.centrodeestudisandaluces.es $>$, documento de trabajo.

GorZ, A. (1967). Strategy for Labor. Boston: Beacon Press.

KogAN, I.; UNT, M. (2005). "Transition from school to work in transition economies». European Societies, 7, p. 219-253.

LAWLER, E. L.; PORTER, L. W. (1967). «The effect of performance on job satisfaction». Industrial Relations, 7, p. 20-28.

LENSKI, G. E. (1967). "Status inconsistency and the vote: a four nation test». American Sociological Review, 32, p. 298-301.

- (1954). "Status crystalization: a non.vertical dimension of social status». American Sociological Review, 19, p. 405-413.

- (1956). "Social participation and status crystallization». American Sociological Review, 19, p. 458-464.

POLANYI, K. (1976). "La economía como actividad institucionalizada». En: POLANYI, K.; Arensberg, C. M.; PeArson, H.W. Comercio y mercado en los imperios antiguos. Barcelona: Labor, p. 289-315.

POLLMANN-SCHUlt, M.; BuCHEL, M. (2004). "Career prospects of overeducated workers in West Germany». European Sociological Review, 20, p. 311-331.

RubB, S. (2003). "Overeducation in the labour market: A comment and re-analysis of a meta-analysis». Economics of Education Review, 22, p. 621-629.

- (2003). "Overeducation: a short or long run phenomenon for individuals?». Economics of Education Review, 22, p. 389-394.

- (2005). «Overeducation, undereducation, and the theory of career mobility: a comment and a note on underemployment». Applied Economics Letters, 12, p. 115-118.

Rumberger, R. (1981). Overeducation in the U.S. Labour Market. Nueva York: Praeger. SÁNCHEZ CARrión, J. J. (1995). Manual de análisis de datos. Madrid: Alianza.

SKOTT, P. (2005). «Fairness as a source of hysteresis in employment and relative wages». Journal of Economics Behavior \& Organization, 12, p. 305-331.

SloAne, P. J. (2003). "Much ado About Nothing? What does the Overeducation Literature Really Tell us?». En: BÜCHEL, F., DE GRIP, A.; MERTENS, A. Overeducation in Europe. Cheltenham (UK): Edward Elgar, p. 11-48.

WALD, S. (2005). «The impact of overqualification on job search». International Journal of Manpower, 26, p. 140-156.

WebER, M. (1979). Economía y sociedad. FCE [1a ed. 1922].

Wolbers, M. H. J.; De GraAf, P. M.; Ultee, W. C. (2001). «Trends in the occupational returns to educational credentials in the Dutch labour market: Changes in structures and in the association?». Acta Sociologica, 44, p. 5-19. 